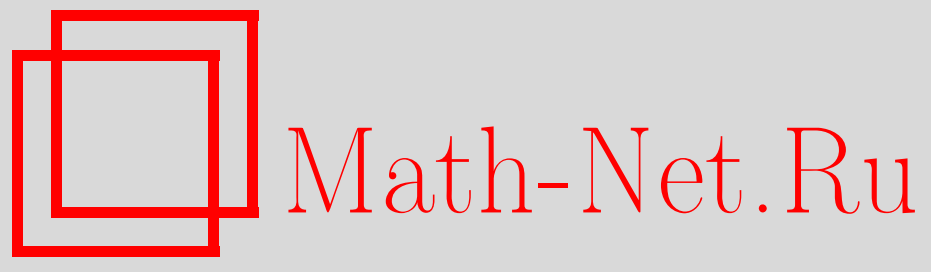

Ш. Р. Шакиров, Старшие дискриминанты бинарных форм, ТMФ, 2007, том 153, номер 2, 147-157

DOI: https://doi.org/10.4213/tmf6133

Использование Общероссийского математического портала Math-Net.Ru подразумевает, что вы прочитали и согласны с пользовательским соглашением http://www . mathnet.ru/rus/agreement

Параметры загрузки:

IP : 3.85 .7 .115

26 апреля 2023 г., 16:15:54

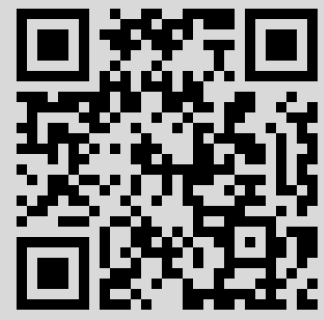




\title{
СТАРШИЕ ДИСКРИМИНАНТЫ БИНАРНЫХ ФОРМ
}

\begin{abstract}
Предложен метод построения систем полиномиальных уравнений, задающих подмногообразия вырожденных бинарных форм с произвольной степенью вырождения.
\end{abstract}

Ключевые слова: дискриминант, вырожденные формы, алгебраические уравнения.

\section{1. ВВЕДЕНИЕ}

Настоящая статья посвящена исследованию вырождения симметрических форм. Известно, что в многообразии всевозможных симметрических форм на $\mathbb{C}^{n}$ вырожденные формы образуют подмногообразие, инвариантное относительно действия структурной группы $S L(n)$. Это подмногообразие задается единственным полиномиальным уравнением $D=0$, где $D$ представляет собой $S L(n)$-инвариантный полином от коэффициентов формы и называется ее дискриминантом.

Данное дискриминантное подмногообразие имеет интересную внутреннюю геометрию: формы с различной степенью вырождения образуют в нем набор подмногообразий, вложенных друг в друга. Естественной задачей является построение систем полиномиальных уравнений, задающих эти подмногообразия. Такие системы уравнений позволяют не только определить, вырожденна ли данная форма, но и выяснить, насколько сильно она вырожденна.

Наиболее общий подход к решению этой задачи описан в статье [1] в терминах представлений группы $S L(n)$. Простейший случай $n=2$ связан с теорией алгебраических уравнений от одной переменной. Данная статья посвящена подробному анализу этого случая.

Однородные симметрические формы и условия их вырождения нередко появляются в физических задачах. В частности, возможным их применением является точное (непертурбативное) вычисление негауссовых производящих функционалов в теории поля. В формализме континуального (функционального) интегрирования

* Институт теоретической и экспериментальной физики, Москва, Россия; Московский физико-технический институт, г. Долгопрудный, Московская обл., Россия.

E-mail: shakirov@itep.ru 
важную роль играют интегралы вида

$$
Z=\int e^{S_{i j} x^{i} x^{j}} d^{n} x=\text { const } \cdot \frac{1}{\sqrt{\operatorname{det} S}},
$$

которые называются гауссовыми интегралами. Они хорошо изучены, поскольку форма $S$ является квадратичной формой, а для квадратичной формы дискриминант $D(S)$ в точности равен определителю формы $\operatorname{det} S$. Теория вычисления негауссовых интегралов (с формой произвольной степени) развита хуже. Весьма вероятно, что она тесно связана с теорией дискриминантов и старших дискриминантов.

\section{2. БИНАРНЫЕ ФОРМЫ И ИХ КОРНИ}

Бинарной $k$-формой называется симметрическая форма степени $k$ на пространстве $\mathbb{C}^{2}$, т.е. однородный полином от двух комплексных переменных

$$
P_{k}(x, y)=a_{k} x^{k}+a_{k-1} x^{k-1} y+a_{k-2} x^{k-2} y^{2}+\cdots+a_{0} y^{k} .
$$

Числа $a_{i}$ называются коэффициентами формы $P$. Поскольку основное поле $\mathbb{C}$ алгебраически замкнуто, то любой такой полином может быть разложен в произведение $k$ линейных множителей:

$$
P_{k}(x, y)=a_{k} x^{k}+a_{k-1} x^{k-1} y+\cdots+a_{0} y^{k}=\left(\alpha_{1} x+\beta_{1} y\right)\left(\alpha_{2} x+\beta_{2} y\right) \ldots\left(\alpha_{k} x+\beta_{k} y\right) .
$$

Отсюда следует, что ядро формы $P$ (множество решений уравнения $P_{k}(x, y)=0$ ) состоит из $k$ одномерных подпространств

$$
\Lambda_{i}=\left\{(x, y) \mid \alpha_{i} x+\beta_{i} y=0\right\} .
$$

Эти прямые называются корнями формы $P$. У бинарной $k$-формы общего вида есть ровно $k$ несовпадающих корней. Вырожденными формами являются те, у которых некоторые корни совпадают друг с другом.

Корни бинарной формы являются одномерными подпространствами в $\mathbb{C}^{2}$, т.е. точками проективного пространства $\mathbb{C} P^{1}$. Тесная связь бинарных форм с обыкновенными алгебраическими уравнениями $k$-й степени состоит в том, что на $\mathbb{C} P^{1}$ можно выбрать некоторую карту, например, $y \neq 0$. Поскольку $\operatorname{dim} \mathbb{C} P^{1}=1$, в этой карте каждый корень $\Lambda_{i}$ представляется одним комплексным числом $\lambda_{i}$. При этом уравнение

$$
P_{k}(x, y)=a_{k} x^{k}+a_{k-1} x^{k-1} y+\cdots+a_{0} y^{k}=0
$$

примет вид

$$
P_{k}(z)=a_{k} z^{k}+a_{k-1} z^{k-1}+\cdots+a_{0}=0
$$

где $z=x / y$ - координата на проективном пространстве в карте $y \neq 0$. Это полиномиальное уравнение $k$-й степени от одной комплексной переменной, которое имеет ровно $k$ корней $\lambda_{i}$. 
Геометрически пространство всех бинарных $k$-форм представляет собой гладкое многообразие $S^{k} \equiv S^{k} \mathbb{C}^{2} \sim \mathbb{C}^{k+1}$, в котором вырожденные формы образуют проективные подмногообразия. Основным среди них является дискриминантная поверхность $S_{2}^{k}$ - множество всех $k$-форм, у которых хотя бы два корня совпадают. Она задается одним полиномиальным уравнением

$$
D_{k}=0
$$

где полином $D_{k}$ называется дискриминантом $k$-формы. Например, для квадратичной формы

$$
P_{2}(x, y)=a x^{2}+b x y+c y^{2}
$$

дискриминант равен

$$
D_{2}(a, b, c)=b^{2}-4 a c
$$

для кубической формы

$$
P_{3}(x, y)=a x^{3}+b x^{2} y+c x y^{2}+d y^{3}
$$

дискриминант есть

$$
D_{3}(a, b, c, d)=-27 a^{2} d^{2}+18 a b c d-4 a c^{3}-4 d b^{3}+b^{2} c^{2} .
$$

Зная дискриминант бинарной формы, мы можем ответить на вопрос, является ли форма вырожденной, т.е. есть ли у нее хотя бы два совпадающих корня. Целью настоящей статьи является построение старших дискриминантов, которые бы к тому же различали степени вырождения, т.е. позволяли установить, сколько корней совпадает у данной формы и с какими кратностями.

На дискриминантной поверхности $S_{2}^{k}$ лежат подмногообразия форм с более высокой степенью вырождения. Например, $S_{3}^{k}$ - множество всех $k$-форм, у которых совпадают хотя бы три корня, или $S_{2,2}^{k}-$ множество всех $k$-форм, у которых совпадают хотя бы две пары корней.

Степень вырождения данной формы определяется разбиением множества ее корней на совпадающие. Для любого разбиения

$$
k=k_{1}+k_{2}+\cdots+k_{p}
$$

где $k_{1} \geqslant k_{2} \geqslant \cdots \geqslant k_{p}$, обозначим $S_{k_{1}, \ldots, k_{p}}^{k}$ множество $k$-форм с таким типом совпадений. В целях упрощения обозначений, если нижний индекс $k_{j}$ в $S_{k_{1}, \ldots, k_{n}}$ равен единице, мы не будем его писать. Например, разбиению

$$
k=1+1+\cdots+1
$$

соответствует пространство всех $k$-форм $S^{k}$, разбиению

$$
k=2+1+\cdots+1
$$

- пространство вырожденных $k$-форм $S_{2}^{k}$ и т.д. 
Необходимо заметить, что разбиение $k=k_{1}+k_{2}+\cdots+k_{p}$ не подразумевает, что группы совпавших корней различны между собой. Например, $S_{4}^{k}$ содержится в $S_{2,2}^{k}$, потому что совпадение четырех корней - это частный случай совпадения двух пар корней. Точно так же $S_{4}^{k}$ содержится в $S_{3}^{k}$, и все подмногообразия содержатся в $S_{2}^{k}$.

Подмногообразие $S_{2}^{k} \subset S^{k}$ задается одним полиномиальным уравнением, но подмногообразия $S_{k_{1}, \ldots, k_{p}}^{k}$ с $k_{1}>2$ имеют меньшую размерность, и поэтому должны задаваться системами из нескольких полиномиальных уравнений. Задача описания таких систем уравнений была поставлена Кэли еще в XIX в., но до сих пор привлекает интерес и все еще исследуется, в том числе самыми современными методами [2].

Группа линейных преобразований $S L(2, \mathbb{C})$

$$
\begin{aligned}
& x \rightarrow G_{11} x+G_{12} y, \\
& y \rightarrow G_{21} x+G_{22} y,
\end{aligned}
$$

где $G_{11} G_{22}-G_{12} G_{21}=1$, естественно действует на симметричные формы на $\mathbb{C}^{2}$. Ясно, что любое подмногообразие вырожденных форм $S_{k_{1}, \ldots, k_{p}}^{k}$, как и дискриминантное подмногообразие $S_{2}^{k}$, инвариантно относительно этого действия, потому что совпадение нескольких прямых на плоскости никак не зависит от выбора базиса в этой плоскости. Поэтому и дискриминант бинарной формы является $S L(2)$-инвариантным полиномом от ее коэффициентов.

Важным свойством подмногообразний вырожденных форм является $S L$-инвариантность, которая позволяет применить к их изучению теорию представлений. Это важно потому, что ядро симметрической формы является совокупностью дискретных прямых только в случае $\mathbb{C}^{2}$. Для $n>2$ произвольная симметричная форма уже не разлагается на множители, другими словами, не существует дискретного набора корней, и вырождение нельзя сформулировать в терминах совпадения корней. Общая теория вырождения симметричных форм, по всей видимости, должна быть сформулирована в теоретико-групповых терминах.

Однако в случае бинарных форм ядро формы представляет собой конечный набор корней, которые могут совпадать друг с другом с различными кратностями. В этом случае можно построить уравнения, задающие формы с высшими степенями вырождения, используя только понятие корней, теорему Виета и математическую логику.

\section{3. ДИСКРИМИНАНТ}

Здесь и далее бинарная форма $P(x, y)$ рассматривается как неоднородный полином $P(z)$ от одной комплексной переменной $z$. Корням формы соответствуют корни алгебраического уравнения $P(z)=0$.

Напомним основные факты об алгебраических уравнениях $k$-й степени от одной комплексной переменной. Такое уравнение имеет вид

$$
a_{k} z^{k}+a_{k-1} z^{k-1}+\cdots+a_{0}=0
$$


и имеет ровно $k$ решений $\lambda_{1}, \lambda_{2}, \ldots, \lambda_{k}$, которые называются его корнями. Корни связаны с коэффициентами формулами Виета

$$
a_{k-i}=a_{k}(-1)^{i} \cdot \sigma_{i}\left(\lambda_{1}, \lambda_{2}, \ldots, \lambda_{k}\right),
$$

где $\sigma_{i}$ обозначает $i$-й элементарный симметрический полином от корней:

$$
\begin{aligned}
& \sigma_{1}\left(\lambda_{1}, \lambda_{2}, \ldots, \lambda_{k}\right)=\lambda_{1}+\lambda_{2}+\cdots+\lambda_{k}, \\
& \sigma_{2}\left(\lambda_{1}, \lambda_{2}, \ldots, \lambda_{k}\right)=\lambda_{1} \lambda_{2}+\lambda_{1} \lambda_{3}+\cdots+\lambda_{k-1} \lambda_{k}, \\
& \sigma_{k}\left(\lambda_{1}, \lambda_{2}, \ldots, \lambda_{k}\right)=\lambda_{1} \lambda_{2} \ldots \lambda_{k}
\end{aligned}
$$

Формулы Виета следуют из разложения

$$
a_{k} z^{k}+a_{k-1} z^{k-1}+\cdots+a_{0}=a_{k}\left(z-\lambda_{1}\right)\left(z-\lambda_{2}\right) \ldots\left(z-\lambda_{k}\right) .
$$

Любой симметрический полином от корней, как известно, представляется в виде полинома от элементарных симметрических полиномов $\sigma_{i}$. Следовательно, любой симметрический полином от корней является функцией от коэффициентов.

Известный способ нахождения дискриминанта уравнения $k$-й степени состоит в том, чтобы рассмотреть функцию от корней вида

$$
D\left(\lambda_{1}, \lambda_{2}, \ldots, \lambda_{k}\right)=\prod_{i<j}\left(\lambda_{i}-\lambda_{j}\right)^{2}
$$

которая является симметрическим полиномом от корней и, следовательно, функцией от коэффициентов. Кроме того, этот полином равен нулю тогда (и только тогда), когда два каких-либо корня совпадают. Эти свойства соответствуют определению дискриминанта. Таким образом, функция $D$ должна совпадать с дискриминантом с точностью до пропорциональности.

Например, для квадратного уравнения $a x^{2}+b x+c=0$

$$
D=\left(\lambda_{1}-\lambda_{2}\right)^{2}=\lambda_{1}^{2}-2 \lambda_{1} \lambda_{2}+\lambda_{2}^{2}
$$

Используя формулы Виета $b / a=-\left(\lambda_{1}+\lambda_{2}\right)$ и $c / a=\lambda_{1} \lambda_{2}$, мы получаем

$$
D=\lambda_{1}^{2}+2 \lambda_{1} \lambda_{2}+\lambda_{2}^{2}-4 \lambda_{1} \lambda_{2}=\left(\frac{b}{a}\right)^{2}-\frac{4 c}{a}=\frac{b^{2}-4 a c}{a^{2}} .
$$

Такой способ построения дискриминанта можно обобщить на любые типы совпадений корней. Необходимо написать выражение от корней, которое было бы равно нулю тогда и только тогда, когда корни совпадают указанным образом (например, совпадают три корня). При этом удобно использовать аппарат математической логики. 


\section{4. СТАРШИЕ ДИСКРИМИНАНТЫ}

Рассмотрим $k$-форму, ее корни $\lambda_{1}, \lambda_{2}, \ldots, \lambda_{k}$ и логическую алгебру $\Lambda$, образованную утверждениями

$$
E_{i j}:\left(\lambda_{i}=\lambda_{j}\right)
$$

с логическими операциями "И" и "ИЛИ", которые мы будем обозначать + и ×, соответственно.

Для любого типа совпадений корней $S_{k_{1}, \ldots, k_{p}}^{k}$ обозначим $L\left[S_{k_{1}, \ldots, k_{p}}^{k}\right]$ логическое выражение, построенное из элементарных утверждений $E_{i j}$, которое является определением этого типа совпадения. Определение строится совершенно естественно для любого типа совпадений. Например, определением дискриминантного подмногообразия $S_{2}^{k}$ является то, что хотя бы одна пара корней совпадает: либо первый корень совпадает со вторым, либо первый корень совпадает с третьим и т.д., таким образом,

$$
L\left[S_{2}^{k}\right]=E_{12} \times E_{13} \times \cdots \times E_{k-1, k}=\underset{i<j}{\times E_{i j}} .
$$

Простыми примерами определений являются

$$
\begin{aligned}
L\left[S_{3}^{3}\right]= & E_{12}+E_{13}+E_{23}, \\
L\left[S_{2,2}^{4}\right]= & \left(E_{12}+E_{34}\right) \times\left(E_{13}+E_{24}\right) \times\left(E_{14}+E_{23}\right), \\
L\left[S_{4}^{4}\right]= & E_{12}+E_{13}+E_{14}+E_{23}+E_{24}+E_{34}, \\
L\left[S_{3}^{4}\right]= & \left(E_{12}+E_{13}+E_{23}\right) \times\left(E_{12}+E_{14}+E_{24}\right) \times \\
& \times\left(E_{23}+E_{24}+E_{34}\right) \times\left(E_{13}+E_{14}+E_{34}\right) .
\end{aligned}
$$

Между логическими утверждениями и уравнениями есть непосредственная связь: для любого утверждения из $\Lambda$ существует эквивалентная ему система уравнений на корни. Например, любому элементарному утверждению $E_{i j}$ соответствует уравнение (для симметричности возведенное в квадрат)

$$
\left(\lambda_{i}-\lambda_{j}\right)^{2}=0 .
$$

Операции "ИЛИ" соответствует умножение операндов, поскольку произведение равно нулю, если равен нулю один из сомножителей. Операции "И" буквально соответствует взятие системы уравнений. Например,

$$
\begin{aligned}
E_{12} & \mapsto\left(\lambda_{1}-\lambda_{2}\right)^{2}=0, \\
E_{34} & \mapsto\left(\lambda_{3}-\lambda_{4}\right)^{2}=0, \\
E_{12} \times E_{34} & \mapsto\left(\lambda_{1}-\lambda_{2}\right)^{2}\left(\lambda_{3}-\lambda_{4}\right)^{2}=0, \\
E_{12}+E_{34} & \mapsto\left\{\begin{array}{l}
\left(\lambda_{1}-\lambda_{2}\right)^{2}=0, \\
\left(\lambda_{3}-\lambda_{4}\right)^{2}=0 .
\end{array}\right.
\end{aligned}
$$

Отметим, что дискриминантному случаю $S_{2}^{k}$ соответствует одно уравнение

$$
L\left[S_{2}^{k}\right]=E_{12} \times E_{13} \times \cdots \times E_{k-1, k} \mapsto \prod_{i<j}\left(\lambda_{i}-\lambda_{j}\right)^{2}=0,
$$


которое упоминалось выше. Совпадению трех корней у 3-формы соответствует система уравнений

$$
L\left[S_{3}^{3}\right]=E_{12}+E_{13}+E_{23} \mapsto\left\{\begin{array}{l}
\left(\lambda_{1}-\lambda_{2}\right)^{2}=0 \\
\left(\lambda_{1}-\lambda_{3}\right)^{2}=0 \\
\left(\lambda_{2}-\lambda_{3}\right)^{2}=0
\end{array}\right.
$$

Логическому выражению

$$
L\left[S_{2,2}^{4}\right]=\left(E_{12}+E_{34}\right) \times\left(E_{13}+E_{24}\right) \times\left(E_{14}+E_{23}\right)
$$

на первый взгляд не соответствует никакая система уравнений. В этом и подобных случаях необходимо раскрывать скобки, пользуясь тем, что логические операции $\times$ и + удовлетворяют обычному распределительному закону

$$
a \times(b+c)=a \times b+a \times c .
$$

При этом определение $L\left[S_{2,2}^{4}\right]$ примет вид

$$
\begin{aligned}
L\left[S_{2,2}^{4}\right]=E_{12} & \times E_{13} \times E_{14}+E_{12} \times E_{24} \times E_{14}+ \\
& +E_{12} \times E_{13} \times E_{23}+E_{12} \times E_{24} \times E_{23}+E_{34} \times E_{13} \times E_{14}+ \\
& +E_{34} \times E_{24} \times E_{14}+E_{34} \times E_{13} \times E_{23}+E_{34} \times E_{24} \times E_{23} .
\end{aligned}
$$

Соответствующая система уравнений есть

$$
\begin{aligned}
& \left(\lambda_{1}-\lambda_{2}\right)^{2}\left(\lambda_{1}-\lambda_{3}\right)^{2}\left(\lambda_{1}-\lambda_{4}\right)^{2}=0, \\
& \left(\lambda_{1}-\lambda_{2}\right)^{2}\left(\lambda_{2}-\lambda_{4}\right)^{2}\left(\lambda_{1}-\lambda_{4}\right)^{2}=0, \\
& \left(\lambda_{1}-\lambda_{2}\right)^{2}\left(\lambda_{1}-\lambda_{3}\right)^{2}\left(\lambda_{2}-\lambda_{3}\right)^{2}=0, \\
& \left(\lambda_{1}-\lambda_{2}\right)^{2}\left(\lambda_{2}-\lambda_{4}\right)^{2}\left(\lambda_{2}-\lambda_{3}\right)^{2}=0, \\
& \left(\lambda_{3}-\lambda_{4}\right)^{2}\left(\lambda_{1}-\lambda_{3}\right)^{2}\left(\lambda_{1}-\lambda_{4}\right)^{2}=0, \\
& \left(\lambda_{3}-\lambda_{4}\right)^{2}\left(\lambda_{2}-\lambda_{4}\right)^{2}\left(\lambda_{1}-\lambda_{4}\right)^{2}=0, \\
& \left(\lambda_{3}-\lambda_{4}\right)^{2}\left(\lambda_{1}-\lambda_{3}\right)^{2}\left(\lambda_{2}-\lambda_{3}\right)^{2}=0, \\
& \left(\lambda_{3}-\lambda_{4}\right)^{2}\left(\lambda_{2}-\lambda_{4}\right)^{2}\left(\lambda_{2}-\lambda_{3}\right)^{2}=0 .
\end{aligned}
$$

Итак, для любого типа совпадений корней изложенный выше метод позволяет построить систему уравнений относительно корней, которая эквивалентна определению этого типа совпадений, следовательно, задает множество форм с таким типом совпадений.

Завершающим шагом является выражение этой системы уравнений через коэффициенты формы. Пусть система уравнений, полученная для некоторого типа совпадений, имеет вид

$$
P_{1}\left(\lambda_{1}, \lambda_{2}, \ldots, \lambda_{k}\right)=0
$$




$$
P_{m}\left(\lambda_{1}, \lambda_{2}, \ldots, \lambda_{k}\right)=0 .
$$

Вообще говоря, полиномы $P_{i}$ не являются симметрическими функциями от корней, следовательно, не могут быть непосредственно представлены как функции от коэффициентов. Эта проблема легко решается симметризацией, то есть переходом к эквивалентной системе

$$
\begin{aligned}
& P_{1}+P_{2}+\cdots+P_{m}=0, \\
& P_{1} P_{2}+P_{1} P_{3}+\cdots+P_{m-1} P_{m}=0, \\
& P_{1} P_{2} \ldots P_{m}=0 .
\end{aligned}
$$

Уравнения этой системы симметричны по полиномам $P_{i}$. Как следствие они симметричны по корням и выражаются через коэффициенты с использованием формул Виета. В результате мы имеем решение поставленной задачи: получена система уравнений на коэффициенты формы, которая задает многообразие форм с данным типом вырождения.

\section{5. СЛУЧАЙ $S_{3}^{3}$}

Для иллюстрации мы рассмотрим условие совпадения трех корней у кубической бинарной формы

$$
P_{3}(x, y)=a x^{3}+b x^{2} y+c x y^{2}+d y^{3}
$$

и соответствующее множество вырожденных форм $S_{3}^{3}$. Определение этого совпадения следующее: все три корня равны друг другу и

$$
L\left[S_{3}^{3}\right]=E_{12}+E_{13}+E_{23} .
$$

Соответствующая система уравнений

$$
\begin{aligned}
& \left(\lambda_{1}-\lambda_{2}\right)^{2}=0, \\
& \left(\lambda_{1}-\lambda_{3}\right)^{2}=0, \\
& \left(\lambda_{2}-\lambda_{3}\right)^{2}=0
\end{aligned}
$$

после симметризации принимает вид

$$
\begin{aligned}
\left(\lambda_{1}-\lambda_{2}\right)^{2}+\left(\lambda_{1}-\lambda_{3}\right)^{2}+\left(\lambda_{2}-\lambda_{3}\right)^{2} & =0, \\
\left(\lambda_{1}-\lambda_{3}\right)^{2}\left(\lambda_{1}-\lambda_{2}\right)^{2}+\left(\lambda_{1}-\lambda_{3}\right)^{2}\left(\lambda_{2}-\lambda_{3}\right)^{2}+\left(\lambda_{1}-\lambda_{2}\right)^{2}\left(\lambda_{2}-\lambda_{3}\right)^{2} & =0, \\
\left(\lambda_{1}-\lambda_{2}\right)^{2}\left(\lambda_{1}-\lambda_{3}\right)^{2}\left(\lambda_{2}-\lambda_{3}\right)^{2} & =0
\end{aligned}
$$

и выражается через коэффициенты $a, b, c, d$ с помощью формул Виета:

$$
\frac{b}{a}=-\left(\lambda_{1}+\lambda_{2}+\lambda_{3}\right),
$$




$$
\begin{aligned}
& \frac{c}{a}=\lambda_{1} \lambda_{2}+\lambda_{1} \lambda_{3}+\lambda_{2} \lambda_{3}, \\
& \frac{d}{a}=-\lambda_{1} \lambda_{2} \lambda_{3} .
\end{aligned}
$$

Мы опускаем несложные преобразования, которые дают следующий результат:

$$
\begin{aligned}
2\left(b^{2}-3 a c\right) & =0, \\
\left(b^{2}-3 a c\right)^{2} & =0, \\
-27 a^{2} d^{2}+18 a b c d-4 a c^{3} & -4 d b^{3}+b^{2} c^{2}=0 .
\end{aligned}
$$

Эта система уравнений эквивалентна определению совпадения трех корней и, следовательно, задает подмногообразие $S_{3}^{3} \subset S^{3}$. Второе уравнение зависимо от первого и может быть опущено.

Необходимо заметить, что эта система не является $S L(2)$-инвариантной, хотя многообразие $S_{3}^{3}$, которое она определяет, инвариантно. Это значит, что преобразованная под действием $S L(2)$ система задает то же самое многообразие. Обозначив через $G$ произвольный элемент $S L(2)$, мы получаем бесконечно много систем вида

$$
\begin{gathered}
2 Z(G)=0, \\
(Z(G))^{2}=0, \\
-27 a^{2} d^{2}+18 a b c d-4 a c^{3}-4 d b^{3}+b^{2} c^{2}=\operatorname{inv}=0,
\end{gathered}
$$

где $Z(G)$ - орбита полинома $b^{2}-3 a c$ под действием группы:

$$
Z(G)=G_{11}^{2}\left(b^{2}-3 a c\right)+G_{11} G_{12}(b c-9 a d)+G_{12}^{2}\left(c^{2}-3 b d\right) .
$$

Любая из этих систем задает многообразие $S_{3}^{3}$ форм с тремя совпавшими корнями.

Интересным фактом является то, что множество совместных нулей всей орбиты $Z(G)$, т.е. множество форм, для которых вся орбита $Z(G)$ равна нулю, в точности совпадает с $S_{3}^{3}$. Это позволяет считать орбиту $Z(G)$ старшим дискриминантом в случае совпадения трех корней из трех. Вся орбита, конечно, является $S L(2)$-инвариантным объектом. С точки зрения теории представлений эта орбита реализует некоторое неприводимое представление группы $S L(2)$ в пространстве квадратичных полиномов от коэффициентов.

\section{6. СЛУЧАЙ $S_{3}^{4}$}

В качестве второго примера построим систему уравнений, определяющую формы четвертой степени

$$
P_{4}(x, y)=a x^{4}+b x^{3} y+c x^{2} y^{2}+d x y^{3}+e y^{4},
$$

которые имеют один корень кратности 3. Определение интересующего нас типа совпадений имеет вид

$L\left[S_{3}^{4}\right]=\left(E_{12}+E_{13}+E_{23}\right) \times\left(E_{12}+E_{14}+E_{24}\right) \times\left(E_{23}+E_{24}+E_{34}\right) \times\left(E_{13}+E_{14}+E_{34}\right)$. 
Используя обычные правила математической логики, несложно проверить, что это определение эквивалентно более простому утверждению

$$
L^{\prime}=E_{12} \times E_{34}+E_{13} \times E_{24}+E_{14} \times E_{23},
$$

которому соответствует система уравнений

$$
\begin{aligned}
& \left(\lambda_{1}-\lambda_{2}\right)^{2}\left(\lambda_{3}-\lambda_{4}\right)^{2}=0 \\
& \left(\lambda_{1}-\lambda_{3}\right)^{2}\left(\lambda_{2}-\lambda_{4}\right)^{2}=0, \\
& \left(\lambda_{2}-\lambda_{3}\right)^{2}\left(\lambda_{1}-\lambda_{4}\right)^{2}=0 .
\end{aligned}
$$

Симметризуя эту систему, мы получаем

$$
\begin{aligned}
&\left(\lambda_{1}-\lambda_{2}\right)^{2}\left(\lambda_{3}-\lambda_{4}\right)^{2}+\left(\lambda_{1}-\lambda_{3}\right)^{2}\left(\lambda_{2}-\lambda_{4}\right)^{2}+\left(\lambda_{2}-\lambda_{3}\right)^{2}\left(\lambda_{1}-\lambda_{4}\right)^{2}=0, \\
&\left(\lambda_{1}-\lambda_{2}\right)^{2}\left(\lambda_{3}-\lambda_{4}\right)^{2}\left(\lambda_{1}-\lambda_{3}\right)^{2}\left(\lambda_{2}-\lambda_{4}\right)^{2}+\left(\lambda_{1}-\lambda_{2}\right)^{2}\left(\lambda_{3}-\lambda_{4}\right)^{2} \times \\
& \times\left(\lambda_{2}-\lambda_{3}\right)^{2}\left(\lambda_{1}-\lambda_{4}\right)^{2}+\left(\lambda_{1}-\lambda_{3}\right)^{2}\left(\lambda_{2}-\lambda_{4}\right)^{2}\left(\lambda_{2}-\lambda_{3}\right)^{2}\left(\lambda_{1}-\lambda_{4}\right)^{2}=0, \\
&\left(\lambda_{1}-\lambda_{2}\right)^{2}\left(\lambda_{3}-\lambda_{4}\right)^{2}\left(\lambda_{1}-\lambda_{3}\right)^{2}\left(\lambda_{2}-\lambda_{4}\right)^{2}\left(\lambda_{2}-\lambda_{3}\right)^{2}\left(\lambda_{1}-\lambda_{4}\right)^{2}=0 .
\end{aligned}
$$

Эта система с помощью формул Виета

$$
\begin{aligned}
& \frac{b}{a}=-\left(\lambda_{1}+\lambda_{2}+\lambda_{3}+\lambda_{4}\right), \\
& \frac{c}{a}=\lambda_{1} \lambda_{2}+\lambda_{1} \lambda_{3}+\lambda_{1} \lambda_{4}+\lambda_{2} \lambda_{3}+\lambda_{2} \lambda_{4}+\lambda_{3} \lambda_{4}, \\
& \frac{d}{a}=-\left(\lambda_{1} \lambda_{2} \lambda_{3}+\lambda_{1} \lambda_{2} \lambda_{4}+\lambda_{1} \lambda_{3} \lambda_{4}+\lambda_{2} \lambda_{3} \lambda_{4}\right), \\
& \frac{e}{a}=\lambda_{1} \lambda_{2} \lambda_{3} \lambda_{4}
\end{aligned}
$$

может быть выражена через коэффициенты формы $a, b, c, d, e$. Вычисление дает следующий результат:

$$
\begin{gathered}
2\left(c^{2}-3 b d+12 a e\right)=0, \\
\left(c^{2}-3 b d+12 a e\right)^{2}=0, \\
D_{4}=0 .
\end{gathered}
$$

В третьем уравнении системы левая часть равна дискриминанту формы четвертой степени. Как и в предыдущем примере, второе уравнение оказалось зависимо от первого и может быть опущено.

Интересным фактом является то, что все уравнения этой системы инвариантны относительно действия группы $S L(2)$. Инвариантный полином $c^{2}-3 b d+12 a e$ называется аполярой. Таким образом, старшим дискриминантом, соответствующим случаю совпадения трех корней из четырех, является система двух инвариантов: дискриминанта и аполяры. 


\section{7. ЗАКЛЮЧЕНИЕ}

Предлагаемый метод позволяет получать системы уравнений, задающих бинарные формы с любым разбиением корней на совпадающие. Идея метода состоит в том, чтобы выбрать на проективном пространстве $\mathbb{C} P^{1}$ карту, в которой корни представляются комплексными числами, а форма - полиномом от одной переменной. Искомая система уравнений строится в пространстве корней, а затем выражается через коэффициенты с использованием симметрических формул Виета.

Нерешенными остаются вопросы о сопоставлении орбит группы $S L(2)$ и условий вырожденности форм, а также об обобщении этого соответствия на случай размерности пространства $n>2$.

Благодарности. Автор благодарен В. Долотину и А. Морозову за чрезвычайно полезные дискуссии. Работа выполнена при частичной поддержке Федерального агентства по атомной энергии РФ, Программы поддержки ведущих научных школ (грант № НШ-8004.2006.2) и РФФИ (гранты РФФИ-Италия № 06-01-92059-КЭ, 07-02-006454).

\section{Список литературы}

[1] V. Dolotin, A. Morozov, Introduction to non-linear algebra, hep-th/0609022.

[2] J. Chipalkatti, On equations defining coincident root loci, math/0110224.

Поступила в редакцию 3.10.2006, после доработки 2.04.2007 